\title{
Cinema: uma ferramenta pedagó- gica e humanista para temas em Saúde-Educação. A experiência do Cinesocial
}

\author{
Cinema: an humanist and pedagogical tool for themes in \\ Health-Education. The experience of CineSocial
}

Joab J. S. Xavier ${ }^{1}$, Nathalie L. S. Dewulf²; Cristiane M. Peres ${ }^{3}$; Gisele C. Barros 3 ; Karina Pfrimer; Claudia S. Nakao'; Francine Leite ${ }^{5}$; Rafael J. A. Silva ${ }^{6}$; Ricardo L. R. Santos ${ }^{7}$; Rosane A. Monteiro ${ }^{1}$; Antonio Ruffino-Netto Antonio C. D. Carvalho ${ }^{8}$

\begin{abstract}
RESUMO
Modelo do estudo: Abordagem qualitativa de pesquisa Objetivo: foi utilizado recurso audiovisual (filmes) para propiciar reflexões, discussões e atualização de professores, alunos e profissionais da saúde em temas sociais pertinentes às áreas da saúde e educação. Metodologia: o projeto foi estruturado em forma de curso de difusão com cinco encontros, entre agosto e dezembro de 2008. Foram abordados temas relevantes em Saúde e Educação (fome, violência escolar, gravidez na adolescência, processo saúdedoença e drogas na adolescência) e foram convidados especialistas em cada temática para debate. 0 instrumento utilizado para captar as percepções dos participantes foi um questionário de 14 questões semi-estruturadas e ao final de cada encontro foi solicitada a entrega de relatório. Foi realizada Análise Temática de Narrativa de material proveniente dos relatórios individuais de reflexão. Resultados: os encontros tiveram a presença de 49 participantes (estudantes de graduação, professores da rede municipal de ensino, profissionais da área da saúde, pós-graduandos e docentes do ensino superior). $\mathrm{Na}$ análise do material narrativo advindo das questões abertas dos relatórios individuais de reflexão foi possível levantar dois blocos de categorias, sendo que o primeiro deles destacou as expectativas que os participantes tinham antes de iniciar o projeto CineSocial e o segundo bloco levantou a opinião dos participantes sobre a relevância do curso. Discussão e Conclusões: o uso do cinema possibilitou a circulação dos conhecimentos, tornou possível a difusão de experiências, corroborou a ideia de que documentários assim como filmes de ficção podem exprimir os conhecimentos orientados pela Ciência. Pensar a arte do cinema não foi a prioridade dessa experiência, a prioridade foi pensar a vida, a sociedade, a escola, a saúde, a exclusão social e temas do cotidiano presentes dentro ou fora das escolas.
\end{abstract}

Palavras-chave: Educação em Saúde. Metodologia. Ciência. Arte. Cinema como assunto. Filmes e vídeos educativos.

${ }^{1}$ Mestre em Ciências Médicas (Saúde na Comunidade), Departamento de Medicina Social da FMRP-USP. ${ }^{2}$ Pós-doutoranda em Ciências Farmacêuticas da Faculdade de Ciências Farmacêuticas de Ribeirão Preto - USP. ${ }^{3}$ Centro de Apoio Educacional e Psicológico da FMRP - USP. ${ }^{4}$ Docente, curso de Nutrição e Metabolismo do Departamento de Pediatria e Puericultura da FMRP USP. ${ }^{5}$ Doutoranda em Saúde Pública, Faculdade de Saúde Pública - USP. ${ }^{6}$ Professor de Ensino Médio da Secretaria Municipal de Educação da Prefeitura Municipal de Ribeirão Preto. ${ }^{7}$ Mestre em Ciências (Saúde na Comunidade), Departamento de Medicina Social da FMRP-USP. ${ }^{8}$ Docente do Departamento de Medicinal Social da FMRP-USP.
Correspondência:

Cristiane Martins Peres Centro de Apoio Educacional e Psicológico (FMRP/USP) Av. Bandeirantes, 3900 - Monte Alegre 14049-900 - Ribeirão Preto/SP

E:mail: cris@fmrp.usp.br

Artigo recebido em 23/03/2011 Aprovado para publicação em 13/09/2011 


\section{Introdução}

O cinema é uma forma de arte, e como tal, parece razoável pensar que deve fazer parte das inúmeras possibilidades de se expressar a condição huma$\mathrm{na}^{1}$ e favorecer a reflexão crítica. $\mathrm{O}$ cinema poderia ser utilizado como um recurso importante para as áreas da saúde e educação. Esta ferramenta como uma das formas de arte, seria por si só, a expressão da humanização.

O uso de filmes como material didático, particularmente no ensino de ciências, teve início da década de 1910 em alguns países europeus. Antes do início da Primeira Guerra Mundial, em 1914, centenas de documentários didáticos já haviam sido produzidos na França. Sequiências de imagens sobre a reprodução animal, sobre ciclos de vida das plantas, explosões vulcânicas ou sobre eclipses solares ajudavam a tornar currículos mais interessantes e explicações mais compreensíveis. ${ }^{2}$

$\mathrm{O}$ cinema passa a ter uma função educadora no século XX, aumentando as possibilidades do concreto, das vivências que em cada pessoa se encontram reduzidas a um pequeno repertório de experiências reais. ${ }^{3} \mathrm{O}$ cinema nos descobre os recantos do mundo [...] o cinema nos faz sair da abstração em que o homem culto costuma viver. Apresenta a vida no que tem de concreto. ${ }^{3}$

O cinema como metodologia de ensino não assume o papel de solução mágica, mas pode ser um colaborador eficaz para aprendizagem. Mais ainda, pode servir para iniciar um processo de mudança; mudança perseguida para uma formação intelectual sensível à condição humana e à realidade social. ${ }^{4}$

E a literatura revela muitas experiências em diversos níveis de ensino, nas quais o cinema serviu como ferramenta pedagógica: no nível do ensino fundamental ${ }^{5,6}$, médio ${ }^{7,8}$, superior ${ }^{9-12}$ e ensino profissional $^{13,14,15}$. Também foram encontradas experiências em outras áreas do conhecimento, como por exemplo, atividades de extensão ${ }^{16}$, ensino de interdisciplinaridade $^{17}$, ética ${ }^{18,19}$ e humanização e formação em saúde. ${ }^{20,1}$ Assim, o cinema pode servir como um recurso educacional para várias áreas do conhecimento.

Este trabalho teve, portanto, o objetivo de relatar uma atividade de extensão de caráter sócio-educacional e científica que procurou estabelecer por meio do cinema, maior interação entre Universidade e comunidade. Por meio do diálogo com professores da rede municipal de ensino, profissionais da saúde, estudantes e docentes universitários, a linguagem cinema- tográfica poderia ser vista como metodologia de ensino, pesquisa e extensão.

\section{Materiais e métodos}

Foram realizadas 5 sessões do Projeto CineSocial, no período de agosto a dezembro de 2008, em forma de curso de difusão denominado "CineSocial: uma metodologia inovadora para temas de saúde e sociedade". As temáticas discutidas nas sessões do curso foram: fome, violência escolar, gravidez na adolescência, processo saúde-doença e drogas na adolescência e, em cada sessão, foram convidados palestrantes para discussão de cada temática.

Além dos temas centrais, na primeira sessão (ato inaugural), foi também debatido o tema "ArteCiência: interlocução com diferentes áreas de expressão do conhecimento humano" com especialistas das áreas da Saúde Pública, Ciências Sociais, Música e Teatro. E para o encerramento, foi debatido o tema "Cinema e Educação" com especialistas das áreas do Cinema, Antropologia visual e História.

Em virtude da natureza do fenômeno por nós investigado optamos por realizar uma pesquisa de cunho qualitativo. Segundo Maykut, Morehouse (1994) ${ }^{21}$, a pesquisa qualitativa visa capturar as atitudes e as falas das pessoas ao compreender que elas descrevem sua interpretação sobre o mundo.

Minayo (1994) ${ }^{22}$ complementa essa definição ao expressar que a pesquisa qualitativa:

[...] trabalha com um universo de significados, motivos, aspirações, crenças, valores e atitudes, o que corresponde a um espaço mais profundo das relações, dos processos e dos fenômenos que não podem ser reduzidos à operacionalização de variáveis.

Ao considerarmos que uma pesquisa qualitativa necessita de um instrumento que possa capturar a linguagem e o comportamento dos participantes ${ }^{21}$, e ao pensar na proposta desse projeto, a técnica de coleta de dados mais adequada para termos acesso aos relatos foi um questionário semi-estruturado (Anexo A) que foi entregue para cada participante e solicitado aos mesmos que respondessem e enviassem relatório constando suas impressões sobre a temática abordada em cada sessão.

Foi realizada Análise Temática de Narrativa ${ }^{23}$ do material proveniente dos relatórios individuais de reflexão. Após a leitura global destes relatórios, as unidades dos textos foram reduzidas gradativamente 
em duas ou três rodadas de séries de paráfrases*. As reduções operam com generalização e condensação de sentido, transportando sentenças em categorias temáticas (de acordo com metodologia apregoada por Jovchelovich, Bauer; 2002). ${ }^{23}$

Os filmes foram exibidos em um anfiteatro Espaço Cultural Capela - localizado nas dependências do campus da USP Ribeirão Preto e equipado com telão e poltronas confortáveis, semelhantes ao de uma sala de cinema. No início de cada sessão os coordenadores do Projeto CineSocial apresentavam o filme, o tema abordado e o(s) especialista(s) que participaria $(\mathrm{m})$ do debate.

Para cada sessão foi solicitado aos participantes que assinassem lista de presença para verificação do interesse pelos temas levantados. Entre o final da exibição de cada filme e a fase de debates foi oferecido coffee-break (pipoca e refrigerante) com a intenção de remeter ao aspecto lúdico do cinema.

A divulgação do Projeto foi realizada por meio do jornal do Campus de Ribeirão Preto da USP, rádio USP, faixas com logo (Figura 1), mala direta de emails para Secretaria Municipal de Educação, entidades estudantis e grupos de cineclubistas da USP.

\section{Resultados}

Na Tabela 1 é apresentado o número de participantes segundo filmes exibidos e relatórios entre- gues em cada sessão de cinema. Observa-se que houve uma diminuição crescente no número de participantes que escreveram os relatórios. Todavia, a freqüência dos participantes inscritos nas sessões se manteve constante, alterando mais a participação esporádica que provavelmente refletiu o fato de determinados temas despertarem maior interesse.

Entre os participantes, como mostrado na tabela 2, pode-se observar que houve a participação de pessoas de diversas áreas do conhecimento. Predominaram alunos de graduação, professores da rede municipal de ensino e profissionais da saúde.

Quanto aos relatórios individuais entregues; a apreensão deste material possibilitou a emergência de dois blocos de categorias, sendo que o primeiro deles (Tabela 3) destacou as expectativas que os participantes tinham antes de iniciar o Projeto CineSocial e o segundo bloco (Tabela 4) levantou a opinião dos participantes sobre a relevância do curso.

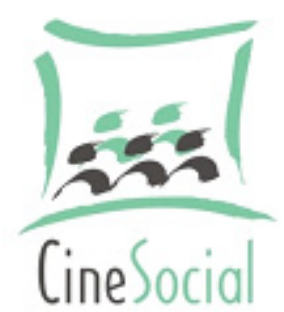

Figura 1: Logomarca desenvolvida para o projeto CineSocial.

Tabela 1

Temas debatidos, participantes por sessão e a entrega de relatórios correspondentes

\begin{tabular}{|c|c|c|c|c|c|}
\hline Filmes & $\begin{array}{c}\text { Tema } \\
\text { debatido }\end{array}$ & $\begin{array}{l}\text { Participantes } \\
\text { do curso }\end{array}$ & $\begin{array}{l}\text { Relatórios } \\
\text { individuais } \\
\text { entregues }\end{array}$ & $\begin{array}{l}\text { Participações } \\
\text { esporádicas }\end{array}$ & $\begin{array}{c}\text { Total de } \\
\text { Participantes }\end{array}$ \\
\hline Ilha das Flores (curta metragem) & Fome & 14 & 10 & 22 & 36 \\
\hline $\begin{array}{l}\text { Escritores da Liberdade } \\
\text { (longa metragem) }\end{array}$ & Violência escolar & 15 & 11 & 11 & 26 \\
\hline Juno (longa metragem) & Gravidez na adolescência & 14 & 04 & 04 & 18 \\
\hline $\begin{array}{l}\text { Super Size Me } \\
\text { (documentário) }\end{array}$ & Processo saúde-doença & 14 & 05 & 16 & 30 \\
\hline $\begin{array}{l}\text { Consciente irracional e } \\
\text { Violência e drogas na } \\
\text { escola (curtas metragens) }\end{array}$ & Drogas na adolescência & 12 & 04 & 05 & 17 \\
\hline
\end{tabular}

*Paráfrase: s.f. 1. Desenvolvimento do texto de um livro ou de um documento conservando-se as ideias originais; metáfrase. 2. Tradução livre ou desenvolvida. (Dicionário Aurélio Básico da Língua Portuguesa. Ed. Nova Fronteira / Folha de São Paulo. Rio de Janeiro, 1995) 
Tabela 2

Caracterização dos participantes do projeto CineSocial

\begin{tabular}{ll}
\hline Área de atuação & $\mathrm{N}^{\mathrm{o}}$ \\
\hline Estudantes de graduação & 15 \\
Professores rede municipal de ensino & 15 \\
Profissionais da área da saúde & 12 \\
Pós-graduandos & 04 \\
Docentes do ensino superior & 03 \\
Total de participantes & 49 \\
\hline
\end{tabular}

Tabela 3

Expectativas que os participantes tinham em relação ao Projeto CineSocial

Categorias de expectativas em relação ao curso

Interesse pelos temas

Oportunidade de troca de conhecimento entre profissionais de múltiplas áreas

Proporcionar novos olhares

Possibilidade de associar cultura à pratica docente

Tornar o aprendizado mais prazeroso

\section{Tabela 4}

Opinião dos participantes sobre a relevância do Curso de Difusão "CineSocial"

Relevância do curso

Contribuições para a prática educativa

Recurso rico para provocar discussões e trocar experiências

Veículo e instrumento pedagógico que possibilitou aprendizado prazeroso

\section{Discussão e conclusões}

Das cinco categorias de expectativas levantadas (Tabela 3), apresentam-se alguns trechos das falas dos participantes (P) para ilustração de algumas destas expectativas:

\section{Oportunidade de troca de conhecimento en- tre profissionais de múltiplas áreas}

Percebeu-se que os encontros oportunizaram a discussão e a troca de idéias entre profissionais de múltiplas áreas. Por meio dos filmes surgiram espon- taneamente, nas discussões e nos relatórios, comentários sobre aplicação das temáticas nas práticas profissionais específicas de cada participante.

"Apesar de já conhecer o filme, considero que a possibilidade de assisti-lo novamente e de participar do debate ao final da apresentação contribuíram para enriquecer minha formação." (P13)

"Os assuntos a serem tratados pelo curso são do interesse direto da minha área de atuação." (P7)

\section{Proporcionar novos olhares}

Transpareceu a todos que as temáticas propostas e as questões que emergiam não tinham a pretensão de chegar a um lugar comum, ao contrário, era importante o conflito, a multiplicidade e a diferenciação para que a reflexão se estabelecesse.

"Para mim, a sua maior importância foi a de ter instigado uma discussão riquíssima ao final, que me fez pensar de forma diferente acerca da temática abordada, despercebidas anteriormente." (P11)

\section{Tornar o aprendizado mais prazeroso}

O cinema como veículo prazeroso para ampliação e aquisição de conhecimento foi uma categoria que englobou tanto como expectativa quanto relevância do curso. A educação há muito já compreendeu que o pensamento inteligente só poderia resultar de um pensamento desejante ${ }^{24}$ que traduza inteligente por crítico e reflexivo. As sessões se mostraram um caminho para redimensionar as ferramentas que levam ao aprendizado.

"... importante, pois é uma maneira prazerosa de adquirir conhecimento." (P3)

"me senti motivada, após participar deste primeiro encontro, para assistir outros filmes, a me adentrar neste universo ainda pouco explorado por mim. Percebi o potencial de sensibilização do cinema e as suas possibilidades para o aprendizado e reflexões críticas." (P11)

A seguir são apresentados alguns trechos de falas dos participantes para ilustração daquilo que, segundo suas opiniões, foi relevante no curso (Tabela 4).

\section{Contribuições para a prática educativa}

$\mathrm{O}$ curso gerou discussões sobre o uso do cinema na Educação e a necessidade de se incluir esta 
área de estudo na formação dos professores. Foi debatido quanto o domínio do conhecimento sobre a arte cinematográfica poderia permitir o melhor aproveitamento dessa ferramenta no âmbito educacional.

Talvez, esta categoria tenha sido aquela que melhor representou a congruência dos objetivos do projeto, ou seja, tornar a apresentação de filmes um instigador de grande valor para uma prática educativa que seja mais humanizada, reflexiva e efetiva.

"Escolhi fazer esse curso, pois além de gostar muito de cinema, como lazer, acredito que os filmes podem ser utilizados na educação como uma metodologia alternativa, dinâmica e diferenciada." (P2)

"Considero que o cinema pode ser um excelente recurso educacional, desde que o educador apresente formação que lhe permita aproveitar os recursos oferecidos por esta arte." (P13)

\section{Recurso rico para provocar discussões e tro- car experiências}

Este era o momento que, apesar de seu caráter informal, trazia em seu bojo a grande responsabilidade de tornar-se um espaço de acolhimento para as diferentes visões, crenças, profissões e opiniões.

Os organizadores do curso acreditaram também que, "permitir no espaço acadêmico o fluir das emoções - por meio da discussão, de partilhar os sentimentos, abre caminhos para uma verdadeira reconstrução da afetividade". ${ }^{3}$ Ao sentir-se à vontade, a platéia pode se posicionar "cognitivamente e emocionalmente".

"A discussão fomentada após a exibição do filme não deu conta do leque de emoções e reflexões que gerou. Fato notório que seria possível estendê-la muito ainda. Em mim muitas impressões permaneceram..." (P7)

"Considero que o debate foi uma parte igualmente rica no evento e destaco as contribuições preciosas do professor (...) para que pudéssemos desenvolver uma avaliação mais crítica e direcionada do filme. A presença dos professores na platéia também deu um toque especial na dis- cussão ao contextualizar a prática segundo temas abordados no filme (...)." (P11)

O "distanciamento da universidade e o público extra-campus" também se tornou uma temática implícita no decorrer do curso. Ainda, que o projeto, desde a escolha de seu nome e de seu público alvo tivesse o intuito da inclusão de professores de todos os níveis da educação e profissionais da saúde, a maioria dos participantes foi da comunidade USP. Durante o debate de alguns temas houve o questionamento por parte de alguns profissionais da rede municipal sobre a existência de uma disparidade entre o discurso acadêmico e a realidade do contexto educacional.

O cinema pode ser um recurso valioso para motivar, envolver e "tirar do lugar comum" a prática da sala de aula; tem utilidade na educação como uma metodologia alternativa, dinâmica e diferenciada; contribuindo para motivação do aluno. Ainda que assuma o papel do entretenimento torna-se uma ferramenta de reflexão espontânea que permite ao espectador analisar criticamente algumas situações, apropriar-se das produções e das linguagens artísticas, emocionarse, atualizar-se, divertir-se.

O uso do cinema possibilitou a circulação do conhecimento, tornou possível a difusão de experiências, re-visitar temáticas com olhares individuais que, ao serem expostas à reflexão coletiva, puderam ser reelaboradas.

Concluiu-se que pensar o cinema não foi a prioridade dessa experiência, a prioridade foi pensar a vida, a sociedade, a escola, a saúde, a exclusão social, os temas do cotidiano que nos deparamos dentro ou fora da escola e da Universidade.

Como proposto no projeto original, foram adquiridos os filmes apresentados nas sessões, assim como outros filmes de temas relacionados à área da Saúde Pública e Educação e após a finalização do projeto, quinze filmes - "O jardineiro fiel", "Anjos do sol", "Menina de ouro", "Patch Adams - o amor é contagioso", "Três irmãos de sangue", "Sicko - SOS Saúde", "Doutores da Alegria - o filme", "O risco sempre existe (VHS)", "Escritores da Liberdade", "Juno", "Super Size Me", "Curtas da Casa de Porto Alegre" (três DVDs) e "Um dia de vida" foram doados para a criação de uma videoteca, instalada nas dependências do Departamento de Medicina Social da FMRP/USP. 


\begin{abstract}
Study design: The approach to the research was qualitative. Objective: we used the audiovisual resources (movies) to make teachers, students and health professionals reflect, discuss and update social subjects relevant to health and education. Methodology: The Project was structured as a course with five meetings that took place between 2008 August and December. Subjects relevant to Public Health and Education were focused (hunger, school violence, teenage pregnancy, health-disease process and teenage drug abuse); for each theme, specialists were invited. The instrument used to acquire the participant perceptions was a questionnaire with 14 semi-structured questions and at the end of each meeting, a report was requested. A Thematic analysis of narrative was conducted with the material from the individual reflection report. Results: The meeting had the presence of 49 participants (undergraduates, public teachers, health professionals, graduates and professors). In the analysis of the narrative open question material from the individual reflection report, we could identify two sets of categories: the first one identified was the participants' expectancy before beginning the CineSocial project and the second one identified was the participants' opinions about the course relevance. Discussion and Conclusions: The cinema use enabled knowledge flow and experience diffusion; it also corroborated the idea that not only documentaries and fiction films could express the knowledge aimed by Science, but other types of cinema as well. Focusing on cinema was not the priority of this experience, the priority was focus on life, society, school, exclusion, current subjects present inside or outside of the school.
\end{abstract}

Keywords: Health Education. Methodology. Science. Art. Motion Pictures as Topic. Instructional Films and Videos.

\section{Agradecimentos}

Este projeto obteve apoio do Fundo de Fomento às Iniciativas de Cultura e Extensão da Pró-Reitoria de Cultura e Extensão Universitária da USP. Os membros do projeto agradecem ao apoio financeiro e logístico do Departamento de Medicina Social da FMRP/USP, ao Thiago Souto pela elaboração do logo do Projeto CineSocial e à Márcia Baumann Di Stasio, do Centro de Apoio Educacional e Psicológico da Faculdade de Medicina de Ribeirão Preto da Universidade de São Paulo (CAEP/FMRP-USP) pelo suporte administrativo.

\section{Referências bibliográficas}

1. Blasco PG. É possível humanizar a medicina? Reflexões a propósito do uso do cinema na educação médica. O mundo da saúde 2010; 34: 357-67

2. Oliveira BJ. Cinema e imaginário científico. Hist Cienc SaudeManguinhos 2006; 13 (suplemento): 133-50.

3. Marias J. La educación sentimental. Madrid: Alianza Editorial, 1992. 228p.

4. Blasco PG. Medicina de Família \& Cinema: Recursos Humanísticos na Educação Médica. Casa do Psicólogo. 2002. p.76-86. 312p.

5. Fantin M. Mídia-Educação e cinema na escola. Rev Teias 2007; 8: $10-23$

6. Paula ME, Chaves EM. Crianças passeiam pelas veredas do cinema, por meio da tecnologia. Rev Teias 2007; 8: 1-9

7. Abud KM. A construção de uma Didática da História: algumas idéias sobre a utilização de filmes no ensino. História 2003; 22: 183-93.

8. Piassi LP, Pietrocola M. Ficção científica e ensino de ciências: para além do método de "encontrar erros em filmes". Educ Pesqui 2009; 35: 525-40.

9. Blasco PG, Gallian DMC, Roncoletta AFT, Moreto G. Cinema para o Estudante de Medicina: um Recurso Afetivo/Efetivo na Educação Humanística. Rev Bras Educ Med 2005; 29: 119-28.

10. Ventura $S$, Onsman A. The use of popular movies during lectures to aid the teaching and learning of undergraduate pharmacology. Med Teach 2009; 31: 662-4.

11. Brown ST, Kirkpatrick MK, Magnum D, Avery J. A review of narrative pedagogy strategies to transform traditional nursing education. J Nurs Educ 2008; 47: 283-6.

12. Maia JMC, Castilho SM, Maia MC, Lotufo Neto F. Psicopatologia no cinema brasileiro: um estudo introdutório Rev psiquiatr clin 2005; 32: 319-23.

13. Rabow MW, Goodman S, Chang S, Berger M, Folkman S. Filming the family: a documentary film to educate clinicians about family caregivers of patients with brain tumors. J Cancer Educ 2010; 25: 242-6.

14. Jukic V, Brecic P, Savic A. Movies in education of psychiatry residents. Psychiatr Danub 2010; 22: 304-7.

15. Datta V. Madness and the movies: an undergraduate module for medical students. Int Rev Psychiatr 2009; 21: 261-6.

16. Meneghel SN. O Homem Elefante: reflexões sobre saúde, doença e anormalidade Interface Comunic Saude Educ 2008; 12: 427-32.

17. Peterson TE. The Art of Language Teaching as Interdisciplinary Paradigm. Educ Philos Theor 2008; 40: 900-18.

18. Saab BR, Sidani N, Merheb M, Mahmassani D, Ghaddar F Hamadeh G. Zooming in to Health Ethics: An Action to Promote Ethics. Fam Med 2009; 41: 17-21.

19. Fischer RMB. Docência, cinema e televisão: questões sobre formação ética e estética. Rev Bras Educ 2009; 14: 93-102.

20. Archanjo LR, Fraiz IC. Medcine: o cinema como recurso humanístico na formação profissional. RUBS 2006; 2: 43-48

21. Maykut $P$, Morehouse R. Beginning qualitative research: a philosophic and practical guide. London: Falmer Press, 1994.

22. Minayo MCS (org.). Pesquisa social: teoria, método e criatividade. Petrópolis, RJ: Vozes. 1994.

23. Jovchelovich S, Bauer MW. Entrevista narrativa. In: Bauer MW, Gaskell G. Pesquisa qualitativa com texto, imagem e som - um manual prático. Petrópolis: Editora Vozes, 2002. p 90-113.

24. Fernández A. A inteligência aprisionada. Porto Alegre: Artmed Editora, 1991. 261p. 


\section{ANEXOA}

\section{UNIVERSIDADE DE SÃO PAULO \\ FACULDADE DE MEDICINA DE RIBEIRÃO PRETO PROJETO CINESOCIAL}

Av Bandeirantes, 3900. Monte Alegre. Ribeirão Preto. SP. CEP 14049-900

Tel 16.3602.3195

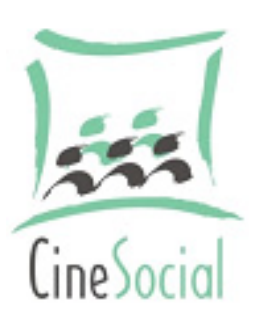

\section{ROTEIRO PARA ELABORAÇÃO DE RELATÓRIO}

\section{SUGESTÕES PARA REALIZAR O CURSO}

- Basta ler as questões, para tomar conhecimento do projeto. Não se preocupe em guardá-las nem memorizálas. Preserve a espontaneidade sem se ater a regras, ou pensando que é uma tarefa a cumprir.

- Guarde as cenas - diálogos, tomadas de câmera, ou circunstâncias quaisquer - do filme que despertaram seu interesse. Isso facilitará a localização posterior quando você mesmo, relendo este questionário no fim, analisar suas próprias experiências e as analisar.

\section{QUESTIONÁRIO}

\section{Roteiro para reflexão individual}

1. Por que estou inserido neste curso? Qual foi o interesse que me fez participar? (Para apenas o primeiro questionário)

2. O tempo dedicado para ver um filme considero importante, ou "concorrente" com outras prioridades imediatas (tempo de estudo, descanso, preparação de provas, trabalhos, etc)?

3. Qual o significado geral - sintético - do filme que vi? Tem importância para mim? Qual? Por quê?

4. Que atitudes - das personagens, situações ou descrições - me surpreenderam?

5. São úteis como modelo? São aplicáveis à minha realidade? Por quê?

6. E as atitudes que me desafiam, das quais não me sinto capaz? O que me falta?

7. Encontrei modos de dizer ou recursos para exprimir realidades que não sabia explicar antes? Teve alguma utilidade ver esse filme para minha profissão? Qual?

8. As circunstâncias e atitudes expostas me fizeram lembrar alguém que me é próximo: familiar, amigo, colega, conhecido? Por quê? (resposta optativa para o relatório por escrito)

9. No dia-a-dia lembrei-me de cenas vistas enquanto desempenhava as atividades cotidianas? Se assim foi, o que motivou essa vocação?

10. Que atitudes das personagens, situações ou descrições me surpreenderam? Comente.

11. O filme trouxe algum significado para minha formação? Se sim, é possível estabelecer relação ou diálogo entre o(s) filme(s) e minha profissão ou formação? Comente.

12. O filme e o debate ajudaram a sedimentar os conhecimentos apresentados?

13. Senti interesse por ver outros filmes após participar neste projeto? Por que motivo?

14. Que outros filmes recomendaria para incluir no projeto?

Material elaborado com base no livro: Medicina de Família \& Cinema: Recursos Humanísticos na Educação Médica. Pablo Gonzáles Blasco. Casa do Psicólogo. 2002.

Referência complementar: OMS. Preparación de los pofessionales de la atención de salud para el siglo XXI, 2005. 